\title{
The importance of inpatient hypoglycaemia: impact, prediction and prevention
}

Running title: Inpatient hypoglycaemia

Authors:

Yue Ruan ${ }^{1}$, Garry D. Tan ${ }^{1,2}$, Alistair Lumb ${ }^{1,2}$, Rustam D. Rea ${ }^{1,2}$

${ }^{1}$ Oxford Centre for Diabetes, Endocrinology and Metabolism, Oxford University Hospitals NHS Foundation Trust, Churchill Hospital, Oxford OX3 7LE, United Kingdom

${ }^{2}$ NIHR Oxford Biomedical Research Centre, Oxford University Hospitals NHS Foundation Trust, Oxford OX3 9DU, United Kingdom

Corresponding author:

Rustam Rea

Oxford Centre for Diabetes, Endocrinology and Metabolism

University of Oxford, Oxford, United Kingdom

tel: +44, email: rustam.rea@nhs.net

Word count:

Main text

Abstract:

Figures:

Tables:

References:

49

Supplemental online material: 0 


\begin{abstract}
Hypoglycaemia is a key barrier to achieving euglycaemic control for hospitalized patients. Inpatient hypoglycaemia has been implicated to be associated with adverse clinical outcomes, including increased mortality and increased length of stay. A number of studies have applied mathematical tools and statistical models to predict inpatient hypoglycaemia and identify factors that may result in hypoglycaemic events. Several different approaches have been tested to prevent inpatient hypoglycaemia. These can be categorised into human intervention, computerised methods and application of medical devices. This review will provide an overview of the epidemiology of inpatient hypoglycaemia and its impact on patients and hospitals. It will also discuss the existing methodology used to predict inpatient hypoglycaemia and the limited number of trials performed to prevent inpatient hypoglycaemia. The paper highlights the urgent need for evidence-based methods to reduce inpatient hypoglycaemia.
\end{abstract}




\section{Novelty Statement}

- We identified 4,572 references in the literature about the prediction, prevention and management of inpatient hypoglycaemia, but only 49 papers were directly relevant to this review

- Whilst several papers show an association between hypoglycaemia and subsequent negative clinical and economic impact, there is no evidence that this impact can be reversed by preventing inpatient hypoglycaemia

- Several papers have identified multiple predictors of hypoglycaemia through multivariate regression modelling. However, no prospective model has been tested to give an individual prediction of hypoglycaemia at the point of admission, enabling preventative strategies to be employed

- Prospective predictors of inpatient hypoglycaemia and the development of evidence-based tools to prevent hypoglycaemia should be the purpose of future research 


\section{Background}

Hypoglycaemia, defined as blood glucose $\leq 3.9 \mathrm{mmol} / \mathrm{L}$, is an important and common clinical problem in people with diabetes who are hospitalised (see Figure 1 for the prevalence of inpatient hypoglycaemia in England and Wales from the 2017 National Diabetes Inpatient Audit [NaDIA] report). The NaDIA report shows that although the prevalence of all hypoglycaemic episode types has decreased since 2016, almost one in five people with diabetes still experience an episode of hypoglycaemia during their hospital stay.

One of the commonest causes of inpatient hypoglycaemia is the treatment of hyperglycaemia [4]. The current management of inpatient hyperglycaemia in a noncritical care setting remains poor, with glycaemic outcomes varying widely between different centres [1, 2] despite the availability of clear clinical guidance [3]. A recent meta-analysis reported that intensive glycaemic control (with a target glucose of 5.6$10.0 \mathrm{mmol} / \mathrm{L}$ ) in non-critical care patients is associated with a trend to an increased risk of hypoglycaemia [4]. It is estimated that each person with diabetes or hyperglycaemia who experiences hypoglycaemia, does so on 2 occasions on average during their hospitalisation, the majority occurring overnight [5]. Poor knowledge of insulin therapy among healthcare providers and unexpected nutritional interruption coupled with a failure to adjust the patient's insulin therapy contributes to the prevalence of inpatient hypoglycaemia [6]. Training staff to manage variable rate intravenous insulin infusions ('insulin sliding scales'), as well as to understand the often complex insulin regimens used to optimise glucose control, is an ongoing challenge in many centres due to reduced staffing levels and increased workload [6]. All these factors contribute to the high risk of hypoglycaemia amongst inpatients.

There is increasing evidence that hypoglycaemia during an inpatient stay is associated with adverse outcomes. Inpatient hypoglycaemia is widely recognised as a poor prognostic marker in terms of morbidity and mortality, increased length of stay [7] and cost to the healthcare system [8]. The psychological consequences to each person with diabetes experiencing inpatient hypoglycaemia have not been systematically evaluated and correlated against behaviour change and risk of longer term diabetes-related complications. 
This article aims to provide an overview of the epidemiology of inpatient hypoglycaemia and its impact on patients and hospitals. It will also discuss the existing methodology used to predict inpatient hypoglycaemia and the limited number of trials that have tried to prevent inpatient hypoglycaemia. The paper highlights the urgent need for evidence-based methods to reduce inpatient hypoglycaemia.

The databases used for the literature search included Medline, Embase and the Cochrane Library. We looked for papers using key words "diabetes", "hypoglycaemia", “hospitalised", “inpatient”, “insulin”, “impact”, "prediction”, "prevention”, "non-critically ill”, “comorbidity”, “management" and "glycaemia control”. A total of 4,572 papers were identified in the primary search but only 219 papers were regarded as relevant to the present review. The abstracts and full texts from these articles were reviewed and 49 articles were selected and synthesised into this review (see Figure 2 for a literature selection flow chart).

\section{Epidemiology of inpatient hypoglycaemia}

For the purposes of this review, inpatient hypoglycaemia refers to episodes of hypoglycaemia which are experienced during an inpatient stay, and are caused by the treatment of hyperglycaemia or existing diabetes. People who are admitted to the hospital due to hypoglycaemia are beyond the scope of this paper.

The NaDIA surveys the care of people with diabetes admitted to over 200 hospitals in the England and Wales. Over the last 5 years, data from NaDIA have continued to show a high incidence of hypoglycaemia in people with diabetes during their hospital stay with $18.4 \%$ of inpatients with diabetes experiencing at least 1 episode of hypoglycaemia during the one week prior to the inpatient audit in 2017. Seven percent of inpatients with diabetes experienced severe hypoglycaemia (blood glucose $<3.0 \mathrm{mmol} / \mathrm{L}$ ) and $1.3 \%$ of patients experienced episodes of hypoglycaemia requiring injectable rescue treatment in the seven days prior to the audit (185 inpatients) [9]. According to the 2017 NaDIA report, most people with diabetes who had one or more severe hypoglycaemic episodes had been treated with insulin. $30.4 \%$ of people in hospital with type 1 diabetes experienced hypoglycaemia with a blood glucose below $3 \mathrm{mmol} / \mathrm{L} ; 13.2 \%$ of people with insulin treated type 2 diabetes 
experienced similar blood glucose. In contrast, only $2.5 \%$ of people with type 2 diabetes who were not on insulin (but may have been on sulphonylureas) experienced a severe hypoglycaemic episode below $3 \mathrm{mmol} / \mathrm{L}$.

The NaDIA reports that the highest proportion of severe hypoglycaemic episodes takes place between 0500 and $0859 \mathrm{hrs}$ (27.6\% of all severe hypoglycaemic episodes). Previous studies have also demonstrated that hypoglycaemia in hospitalized patients occurs with greater frequency overnight and a large proportion of the events occur due to anti-hyperglycaemic therapy, within two hours of administration of insulin or insulin secretagogues [10].

In one UK study of 3,361,104 people admitted only to internal medicine wards, $27.4 \%$ had a diagnosis of diabetes. Of those, only 5\% experienced secondary hypoglycaemia (hypoglycaemia developed during their inpatient stay). However a total of $10.2 \%$ patients $(4,754$ out of 46,408 patients) with secondary hypoglycaemia died during their hospital stay, compared with $9.5 \%$ patients $(83,508$ out of 879,032 patients) without hypoglycaemia $(P<0.05)[11]$.

A large retrospective study looking at the national health system in Spain reviewed a total of 5,447,725 people with diabetes who were discharged between January 1997 and December 2010. The prevalence of hypoglycaemia during the hospital stay (ie coded as secondary hypoglycaemia, in other words people who were not admitted with hypoglycaemia) increased from $1.1 \%$ in 1997 to a peak of $3.8 \%$ in 2007 before decreasing to $3.2 \%$ in 2010 . The prevalence of primary hypoglycaemia remained stable across the 13 years at approximately $1.5 \%$ [12].

A more detailed UK study looked at 1,458 capillary blood glucose (CBG) tests performed over a 2-week period in all hospital patients. They identified 148 episodes of hypoglycaemia with the mean hypoglycaemia CBG reported at $3.38(+/-0.44)$ $\mathrm{mmol} / \mathrm{L}$. The study also showed that the distribution of hypoglycaemia varied widely by location: $65.8 \%$ of the hypoglycaemia events took place on medical wards, $6.8 \%$ on surgical wards, and $7.4 \%$ in the emergency department. However, immediate treatment changes following the episode of hypoglycaemia were made in only $13.4 \%$ 
of patients, which may account of the high rates of recurrent hypoglycaemia $(25 \%)$ [13].

Inpatient hypoglycaemia often occurs due to changes in medication. The 2017 NaDIA report shows that almost one third of inpatients with diabetes have a medication error during their hospital stay $(31 \%)$ which is likely to be a significant risk factor for hypoglycaemia. Among these medication errors, inappropriate dosage and timing of insulin or insulin secretagogues can directly lead to increased rates of hypoglycaemia. The use of electronic prescriptions may reduce the prescription errors but in themselves may not change the incidence of management errors such as not adjusting insulin or other medication after the first episode of hypoglycaemia. A comprehensive electronic prescribing system integrated with a decision-making algorithm to advise clinicians to adjust medication according to blood glucose levels is more likely to reduce the incidence of inpatient hypoglycaemia.

\section{Impact of inpatient hypoglycaemia}

\subsection{Clinical impact}

\subsubsection{Mortality}

Inpatient hypoglycaemia has been implicated in the development of adverse clinical outcomes, including increased mortality [14]. One retrospective study in Spain showed that hypoglycaemia (blood glucose $<3.9 \mathrm{mmol} / \mathrm{L}$ ) in 154,510 people with diabetes during their hospitalisation was associated with an increased likelihood of in-hospital mortality (odds ratio [OR] 1.12; 95\% confidence interval [Cl], 1.09-1.15) [12]. Another study from the same country used multivariate/multilinear regression models to demonstrate significant associations between inpatient hypoglycaemia and greater inpatient mortality (OR 1.24, 95\% Cl 1.20-1.28) and a greater likelihood of readmission (OR 1.20, 95\% Cl 1.17-1.23) [11].

In the UK, one study from Birmingham in 2012 retrospectively looked at routinely available electronic data of 6,374 admissions from inpatients with diabetes and showed the adjusted OR of inpatient mortality compared to the group without hypoglycaemia was 1.62 (95\% Cl 1.16-2.27) in the group with blood glucose values of $2.3-3.9 \mathrm{mmol} / \mathrm{L}$; the adjusted OR was 2.05 (95\% Cl 1.24-3.38) in the group with 
blood glucose values $\leq 2.2 \mathrm{mmol} / \mathrm{L}$ [15]. A more quantitative conclusion was given by a study from Turchin et al. in 2009. After retrospectively analysing 4,368 admissions in 2,582 patients with diabetes hospitalized in the general wards, they found that the odds of inpatient death rose threefold for every $0.56 \mathrm{mmol} / \mathrm{L}$ decrease in the lowest blood glucose below $3.9 \mathrm{mmol} / \mathrm{L}$ during hospitalization $(P<0.01)$ [16].

\subsubsection{Cardiovascular outcomes}

In a review paper, Natalia et al. summarised the roles of inpatient hypoglycaemia in serious cardiovascular outcomes in diabetes [17]. In the DIGAMI 2 trial 1253 people with type 2 diabetes or hyperglycaemia who were hospitalized with a suspected acute myocardial infarction (MI) were treated with an intravenous infusion of insulin/glucose aiming for normoglycaemia[18]. In the first 24 hours of admission $2.2 \%$ of people experienced a symptomatic glucose of $<3.0 \mathrm{mmol} / \mathrm{L}$. Although these people were more likely to die of all causes ( $95 \% \mathrm{Cl} 1.2$ to 3.3) and of cardiovascular causes (95\% Cl 1.2 to 3.5), during the 2 year follow up, this hazard ratio was no longer significant when corrected for baseline characteristics including age, weight and comorbidity.

In two further trials of intensive insulin / glucose / potassium regimes for people with an acute $\mathrm{MI}, 0.7 \%$ of people with a prior history of diabetes were hypoglycaemia $(\leq 3.8 \mathrm{mmol} / \mathrm{L})$ at the time of admission and a further $2.0 \%$ had at least 1 hypoglycaemic episode during the first 24 hours of admission. People with hypoglycaemia on admission were twice as likely to die as those with normoglycaemia (glucose between 3.9 and $7.0 \mathrm{mmol} / \mathrm{L}$ ) (HR 2.13, 95\% Cl 1.01 to 4.49). However people experiencing hypoglycaemia during their admission did not have an increased mortality at 30 days after adjustment for cofounders [19].

Very few studies have reported the long term non-fatal cardiovascular outcomes following hypoglycaemia while an inpatient. One report from Iran examined the impact of inpatient hypoglycaemia on the incidence of cardiovascular events during a 24-week study in non-critically ill people with type 2 diabetes. In this study, hypoglycaemic episodes and major cardiovascular events were recorded. They found that $44 \%$ of the patients who had experienced hypoglycaemia (blood glucose $<3.9 \mathrm{mmol} / \mathrm{L}$ ) developed cardiovascular events compared to $16 \%$ of those who did 
not experience any hypoglycaemia $(P=0.001)$. The odds ratio for occurrence of major cardiovascular event following hypoglycaemia was $6.75(\mathrm{Cl}=2.4-18.58)$ [20].

\subsubsection{Other clinical impact}

Other adverse clinical outcomes reported following inpatient hypoglycaemia have included increased seizures, falls and more rapid decline in cognitive dysfunction [21]. A study group in the US showed that older individuals and those with comorbidities or severely ill are more likely to suffer from the adverse consequences of inpatient hypoglycaemia including increased mortality and adverse cardiovascular outcomes [22].

Clinicians and nurses may sometimes consider greater glucose variability as a marker of inadequate glucose control. However, recent studies suggest that hypoglycaemia, as opposed to glucose variability, is a stronger predictor of adverse outcomes such as hospital acquired infections, acute renal failure, death and length of stay, in hospitalized, non-critically ill patients [23] [24].

The adverse clinical outcomes associated with hypoglycaemia are important and need prevention. However, existing literature does not demonstrate that avoidance of hypoglycaemia would necessarily result in improved outcomes. Nor is there good evidence that reducing the length of time spent in hypoglycaemia would prevent adverse clinical outcomes. Furthermore, there are few studies investigating the personal characteristics which would help define which people are more susceptible to hypoglycaemia and which people suffer particular adverse outcomes following hypoglycaemia. Further research is needed to understand the nature of the relationship between inpatient hypoglycaemia and the resultant adverse clinical events. Whether this is associative or causative is not clear and needs to be tested with a clear preventative strategy and measure of longer term outcomes.

\subsection{Economic impact}

Previous economic analysis of clinical studies has shown that inpatients exposed to hypoglycaemia have a significantly longer mean duration of hospital stay compared with unexposed patients: estimated 12.04 vs. 9.90 days $(P<0.001)$, in the 
retrospective Spanish study [12] and 10.75 vs 4.73 days in a UK based study [8]. Evans et al. estimated that there was a $24 \%$ increase in the estimated average admission cost per patient for those who experienced a hypoglycaemic event compared to those who did not $(P<0.0001)[8]$.

Another retrospective study performed by Nirantharakumar et al. in the UK in 2012 analysed 6300 inpatient admissions and showed that length of stay, when compared with those without a recorded hypoglycaemic episode, was 1.51 (95\% Cl 1.35-1.68) times higher in the group with blood glucose values of $2.3-3.9 \mathrm{mmol} / \mathrm{L}$ and 2.33 (95\% $\mathrm{Cl}$ 1.91-2.84) higher in the group with blood glucose values less than $2.2 \mathrm{mmol} / \mathrm{L}$ [15].

Increased inpatient length of stay directly affects the percentage occupation of hospital beds. The NaDIA reports around 1 in 6 hospital beds are occupied by a person with diabetes $(18 \%)$ and 1 in 15 of the total population have diabetes $(7 \%)$. The average cost per bed day is $£ 222$ as estimated by a National Institute for Health and Care Excellence (NICE) 2015 report and $£ 400$ estimated by the UK Department of Health in 2017. Based on a recent dataset collected between 2014 and 2018 in the Oxford University Hospitals, we estimated the additional cost to the hospital due to one hypoglycaemic episode would be approximately $£ 1,200$ based on an additional 3 bed days (unpublished data).

Preventing inpatient hypoglycaemia is therefore economically essential for the NHS as well as vitally important for each individual person with diabetes.

\subsection{Psychosocial impact}

In people with type 1 or type 2 diabetes, hypoglycaemia has been found to be associated with a reduction in quality-of-life, increased fear and anxiety and reduced productivity [25]. Depression also has been shown to be related to number of hypoglycaemic episodes [26].

However, the reported psychosocial impact of hypoglycaemia has only been reported in people with diabetes outside of hospital as there are no specific psychological studies in people who experience inpatient hypoglycaemia. Fear of 
hypoglycaemia in the inpatient settings may promote compensatory behaviours from caregivers in order to avoid hypoglycaemia, such as decreased insulin doses or excess carbohydrate intake, resulting in poor glycaemic control. Episodes of hypoglycaemia while the person is an inpatient may reduce the person's trust in the hospital and in healthcare professionals those caring for them while in hospital.

\section{Prediction of inpatient hypoglycaemia}

A number of studies have applied mathematical tools and statistical models to predict inpatient hypoglycaemia (see table 2).

\subsection{Demographic predictors}

A recent study from Cardiff used data collected from 142 people with diabetes during their admission to hospital to create a hypoglycaemia risk score based on 10 criteria. They used multivariable regression to show that type 1 diabetes, type 2 diabetes on insulin, duration of diabetes of $>10$ years, recurrent hypoglycaemia and hypoglycaemia unawareness were the key drivers in the risk score with the latter two weighting higher than the other factors [27].

Also in the UK, Stuart et al. developed a prediction model from 9584 admissions of people with diabetes using multivariable logistic regression which showed that the occurrence of inpatient hypoglycaemia could be predicted by a number of factors including ethnicity (black and Asian), age ( $>=75$ years) and type of admission (emergency) [28].

One retrospective study in the U.S. analysed 294 hypoglycaemic events that occurred in 181 hospitalized patients with type 1 or 2 diabetes. They showed that age was the major demographic determinant, with elderly people with diabetes having the highest risk of hypoglycaemic events in the hospital [29]. Tobin et al. in the US found that greater weight was an important variable in hypoglycaemia prediction [30] [31].

A large study performed by 9 French university hospitals looked at 811 people with diabetes in hospital. They demonstrated that a previous history of severe 
hypoglycaemia and male gender were the main demographic predictive factors for severe hypoglycaemia (blood glucose $<3.0 \mathrm{mmol} / \mathrm{L}$ ) [32].

\section{2 Biomarker predictors}

The aforementioned U.S. retrospective study [29] showed the albumin level may be an indicator of the severity of hypoglycaemia with a lower albumin predicting a more severe hypoglycaemic event. The Tobin study found that creatinine clearance is also a predictive variable of hypoglycaemia [30,31]. The model developed by Stuart et al. identified estimated GFR, C-reactive protein, sodium and albumin as factors in predicting inpatient hypoglycaemia [28]. In these studies, biochemical factors that were not found to be predictive included hyperkalaemia, elevated neutrophil count, haemoglobin and anaemia.

\subsection{Medication predictors}

Tobin et al. identified several medication variables related to a blood glucose $<3.9$ $\mathrm{mmol} / \mathrm{L}$ cut off point including basal, prandial, and adjustable scale insulin doses, and sulfonylurea use [30][31]. Stuart et al. developed confirmed inpatient hypoglycaemia could be predicted by medication (type of insulin and use of sulfonylureas) [28].

\subsection{Comorbidity predictors}

The admission level of $\mathrm{HbA} 1 \mathrm{c}$ has also been shown to be a predictor of the number of hypoglycaemic events (<3.9 mmol/L) in non-ICU patients with T2D [33].

\subsection{Other potential predictors}

Despite the predictors of inpatient hypoglycaemia that have been documented (see Table 2), many other factors related to inpatients hypoglycaemia are not electronically captured (see Table 3 ) during the hospital stay. These include quantity and type of food eaten, amount of exercise performed during the hospital stay and missed meals due to procedures / operations / investigations. The food intake of each person varies, often depending on their cognitive state as well as on their underlying reason for admission. Common causes of inpatient hypoglycaemia are carbohydrate-insulin mismatch and inappropriate dosing of glucose-lowering 
agents. A number of statistical methodologies can be used to mitigate this problem of missing data.

A common theme in the risk prediction models is the link between the dose of insulin and sulphonylureas and the frequency of hypoglycaemia. An important clinical question when moving from prediction to prevention is how to 'smart-dose' the patient when there is a high risk of developing hypoglycaemia without inducing hyperglycaemia.

\section{Prevention of inpatient hypoglycaemia}

Different approaches have been tested to prevent inpatient hypoglycaemia. These can be categorised into human intervention, computerised methods and application of medical devices (Table 4).

\subsection{Human approach}

In a review paper, researchers highlighted that acute illness, hospital routine, and gaps in quality care predispose patients to hypoglycaemia [34]. They highlighted that many of these factors could be minimized when approached from a systems-based perspective. This requires the creation of a multidisciplinary team, including nurses, healthcare professionals and researchers, to develop strategies to prevent hypoglycaemic events. These teams would target many factors, such as systematic analysis of blood glucose measurements, policies and protocols, coordination of nutrition and insulin administration, transitions of care, staff and patient education, and communication [34].

Among the different roles under hospital settings, the role of ward nurses is vitally important in the detection, treatment, and prevention of hypoglycaemia as they are often the first people to be alerted to hypoglycaemia by clinical review of the patient or through the routine bedside monitoring of blood glucose [35]. In one Italian hospital a nurse-managed protocol focusing on carbohydrate intake successfully reduced the incidence of hypoglycaemia in 350 patients with diabetes receiving subcutaneous insulin from $0.34 \pm 0.33$ hypoglycaemic events/day to $0.19 \pm 0.30$ [36]. The protocol included either giving extra carbohydrate, reducing prandial insulin 
according to the actual food intake or to give additional intravenous glucose for patients who undergo prolonged fasting.

Other roles in the multidisciplinary team may also be important. A 4-year intervention study in the US showed that with nursing, dietary and patient education regarding insulin-meal match undertaken, hypoglycaemia could be largely prevented in the majority of inpatients ( $>60 \%$ of cases) [37]. The study was a collective effort from nurses, physicians and patients. While nurses were constantly alert and physician orders for anti-hyperglycaemic therapy frequently revised in high risk patients to avoid hypoglycaemia, patient education was also key to preventing recurrent or severe hypoglycaemia. Close coordination of care between the patient, physician and all other healthcare providers helped identify the cause of hypoglycaemia and which steps should be taken to prevent further episodes.

A large, tertiary acute care hospital in the U.S. established a 'Hypoglycaemia Committee'. Its initiative was unique in that every case of severe hypoglycaemia was reviewed by physicians, endocrinologists, and diabetes specialists. This multidisciplinary approach demonstrated a reduction in preventable hypoglycaemic events [38]. Significant resources would be needed to replicate this in the NHS although the NaDIA - Harms audit has just been launched in the UK to identify all people who have suffered a severe hypoglycaemic event while an inpatient.

The human approach has been demonstrated to be effective in reducing inpatient hypoglycaemia but requires significant investment in both diabetes specialist staff and the development of patient and staff education. A single standardised hypoglycaemia prevention protocol is difficult to apply across different hospitals due to variability of patient characteristics, staff expertise and staffing levels, and local governance structures.

\subsection{Computerised approach}

Through retrospective analysis of in-patient data, a number of mathematical models and computer algorithms have been developed to predict individual risk of developing hypoglycaemia during a hospital stay. Accurate prediction could 
potentially help prevent hypoglycaemia by increasing staff awareness to high-risk patients resulting in closer monitoring and amendment in medication doses [39]. A systematic review looked at fourteen studies applying various kinds of clinical support systems in the care of inpatients with diabetes in non-critical settings. It shows these systems, such as a computerised physician order entry system, may have a beneficial effect in inpatient glycaemic control but needs further confirmation [40].

Kilpatrick et al. from the US performed a randomised control trial to determine whether their predictive informatics hypoglycaemia risk-alert system supported by trained nurse responders would reduce the incidence of severe hypoglycaemia in the hospital. The results showed significant decrease by $68 \%$ in the rate of severe hypoglycaemia in alerted high-risk patients versus non-alerted high-risk patients $(3.1 \%$ vs $9.7 \%, P=0.012$ [41]. This was achieved through nurses-physicians changing orders in $40.7 \%$ of the alerted high-risk patients. Orders were changed in only $20.5 \%$ of the non-alerted high-risk patients after an initial blood glucose of less than 5 $\mathrm{mmol} / \mathrm{L}$ had occurred. A change in orders referred to modification consistent with lowering the risk of hypoglycaemia which involved either discontinuing an oral antidiabetic agent, lowering the dose of insulin, or the addition of dextrose-containing fluids. The most common change in orders was a reduction in the total dose of insulin.

Spat et al. from Austria reported on results from their clinical feasibility study testing the prototype of a mobile, tablet-based client-server system for computerized decision and workflow support and demonstrated the system's positive impact on hypoglycaemia prevention. In this study, 30 inpatients with type 2 diabetes on a basal bolus regime participated in the trial and only $1.3 \%$ of the total blood glucose measurements were $<3.9 \mathrm{mmol} / \mathrm{L}$. The system, which included a computerised decision tool for changing the doses of insulin as well as improving the workflow for decision making, was able to support health care professionals deliver a basal-bolus insulin regimen safely [42].

The computerised approaches to reducing hypoglycaemia are easier to deliver consistently across large hospitals compared to the human approach. They also 
require less investment in training and education. However, the predictive accuracy of the models is limited as underlying between-patient variability is not taken into account. Increasing the amount of clinical data which could be entered into these algorithms, including demographic features, vital signs, medicine administration information and detailed description about their hypoglycaemia episodes, may enable the development of more reliable and personalised hypoglycaemia prevention algorithms. These algorithms will not only need to reduce hypoglycaemia, but also avoid a resultant increase in hyperglycaemia.

\subsection{Medical device approach}

Different medical devices have been used in inpatient settings for management of glycaemic levels in people with diabetes. Apart from the commonly used finger-prick capillary blood glucose testing devices, different real-time continuous glucose monitors, insulin pumps, and closed-loop insulin delivery systems (or 'artificial pancreas') have been under extensive research in the past decade and have been tested in inpatients with diabetes to maintain euglycaemia and to prevent hypoglycaemic episodes.

\subsubsection{Continuous glucose monitoring and insulin pumps}

Continuous glucose monitor (CGM) is a medical device involving insertion of a glucose sensor in the subcutaneous tissue of users. The sensor enables real-time glucose level readings from every 1 to $15 \mathrm{~min}$. An insulin pump is a medical device that continuously titrates insulin, often subcutaneously, into users through an insulin infusion set. The 24-hour infusion rate of insulin can be pre-set in the pump by health care professionals or users themselves for optimal control of glycaemic levels.

A pilot study in the US assessed the feasibility of using a real-time CGM to discontinue subcutaneous insulin pump therapy when hypoglycaemia was predicted in people with type 1 diabetes under controlled conditions in a clinical research facility. Using a linear prediction algorithm with a $4.4 \mathrm{mmol} / \mathrm{L}$ threshold and a $45-\mathrm{min}$ prediction horizon, hypoglycaemia was prevented $80 \%$ of the time [43]. Another

research group from the same country developed subject-specific hypoglycaemia prediction models using time-series analysis on CGM data which have been 
validated against real patient datasets [44]. Neither study was performed in a traditional inpatient setting.

Cameron et al. developed a predictive hypoglycaemia detection algorithm using CGM data with explicit certainty measures to enable early corrective action. The algorithm used multiple statistical linear predictions with regression windows between 5 and 75 minutes and prediction horizons of 0 to 20 minutes [45]. The algorithm has been tested against real inpatient dataset and demonstrated its effectiveness in providing alarms for early corrective actions.

Spanakis et al. used CGM in insulin treated general medicine patients with type 2 diabetes and the results were transmitted to a central nursing station monitoring system. An alarm system was set up at glucose $<4.7 \mathrm{mmol} / \mathrm{L}$. The system successfully prevented any CGM glucose value $<3 \mathrm{mmol} / \mathrm{L}$ in five studied inpatients [46].

\subsubsection{Closed-loop insulin delivery}

A closed-loop insulin delivery system, or artificial pancreas, is a medical device comprising a CGM device, an insulin pump and a control algorithm that is able to instruct optimal rates of real-time insulin infusion from the pump, using blood glucose measurements data collected from the CGM device. The control algorithm is developed to maintain euglycaemia with a pre-defined glucose target level or target range.

Closed-loop control of blood glucose levels in people with type 1 diabetes in an inpatient setting has been demonstrated to be able to increase the time of glucose levels in the target range and to significantly reduce time in hypoglycaemia [47].

Closed-loop insulin delivery without meal-time boluses has also been demonstrated to be safe and effective in insulin-treated adults with type 2 diabetes in the inpatient settings with an increase of $20 \%$ time in the target glucose range. However the system was not designed to reduce hypoglycaemia in the inpatient population and so 
similar time was spent in hypoglycaemic range in people using the closed-loop insulin delivery versus those who were not [48-50].

Closed-loop insulin delivery has been demonstrated to be safe and effective but costly to use in the inpatient settings. A step of pre-selection of patients who would benefit the most from receiving such treatment would be necessary.

\section{Conclusion}

Hypoglycaemia is a common barrier to achieving euglycaemia in the inpatient setting and is associated with significant adverse clinical outcomes for both the person with diabetes and the healthcare system. Hypoglycaemia is important to predict, but existing prediction methods are often limited by the prediction accuracy, with receiver-operating curve analysis reported as an area under the curve of 0.73 [28]. In other studies, the failure rate to predict the hypoglycaemic events in the original analysis was as high as $40 \%$ [28]. These suggests that a predictive equation is only one tool among many that will need to be developed to protect people with diabetes from hypoglycaemia in hospital.

Furthermore, any prediction models and preventative strategies would need to be tested in a range of clinical settings to show that the subsequent reduction in hypoglycaemia and improvement in the clinical outcomes could be generalised between settings. It is also important that prevention of hypoglycaemia does not come at the expense of an increase in hyperglycaemia.

Technology can be helpful in reducing the time spent in hypoglycaemia during an inpatient stay, but it is unlikely to be the only factor in reducing inpatient hypoglycaemia. It is of paramount importance to continue to educate junior doctors and support nursing staff to reduce prescribing and glucose management errors. Doctors and nurses should be aware of the consequences of hypoglycaemia while treating hyperglycaemia.

With large amounts of clinical data available and increasing awareness of the tools of big data analysis, more hypoglycaemia prediction models with greater accuracy can be created and tested. Future research should continue to focus on the 
development of novel computerised drug delivery algorithms and medical devices for the prevention of inpatient hypoglycaemia. Well-designed studies should evaluate the long term clinical outcomes, economic benefit and change in psychological wellbeing when trialling preventative strategies. 
Table 1. Negative impact of inpatient hypoglycaemia

\begin{tabular}{|c|c|c|c|}
\hline & Clinical & Economics & Psychological \\
\hline $\begin{array}{l}\text { Potential } \\
\text { negative } \\
\text { impact }\end{array}$ & $\begin{array}{ll}\text { - } & \text { Increased } \\
& \text { mortality[12] } \\
- & \text { Increased } \\
\text { likelihood of } \\
\text { readmission[11] } \\
-\quad \text { Increased } \\
\text { comorbidities } \\
\text { (cardiovascular } \\
\text { event [51]) } \\
- \text { Other adverse } \\
\text { outcomes } \\
\text { (seizures, falls, } \\
\text { cognitive } \\
\text { dysfunction } \\
\text { [21]) }\end{array}$ & 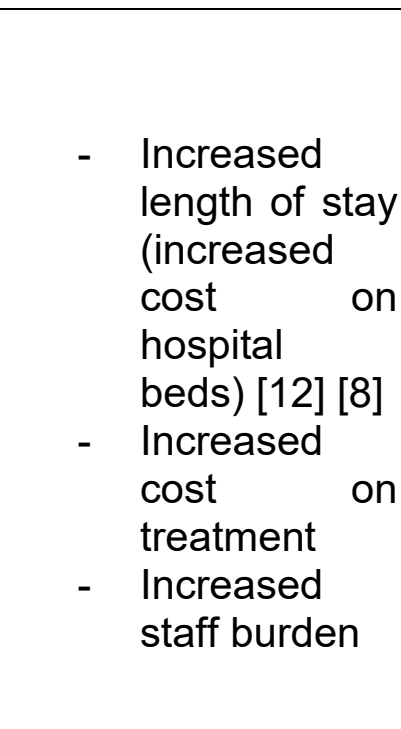 & $\begin{array}{ll}\text { - } & \text { Increased fear and } \\
& \text { anxiety[25] } \\
\text { - } & \text { Reduced quality-of- } \\
& \text { life[25] } \\
\text { - } & \text { Depression[26] }\end{array}$ \\
\hline
\end{tabular}

Table 2. Predictors of inpatient hypoglycaemia

\begin{tabular}{|c|c|}
\hline $\begin{array}{l}\text { Category of } \\
\text { predictors }\end{array}$ & Predictors \\
\hline Demographics & $\begin{array}{ll}\text { - } & \text { age greater than } 75 \text { years old [29] [28] } \\
\text { - } & \text { sex (Male)[32] } \\
\text { - } & \text { weight (higher)[30] [31] } \\
\text { - } & \text { ethnicity (Black and Asian)[28] } \\
\text { - } & \text { type of diabetes (Type 1)[27] } \\
\text { - } & \text { duration of diabetes[27] } \\
\text { - } & \text { hypoglycaemia unawareness [27] }\end{array}$ \\
\hline Drugs & $\begin{array}{l}\text { - Insulin[27] [30] [31] [28] } \\
\text { - } \quad \text { sulfonylurea[27] [30] [31] [28] }\end{array}$ \\
\hline Other & $\begin{array}{l}\text { - HbA1c on admission[33] } \\
\text { - } \text { albumin level less than } 35 \mathrm{~g} / \mathrm{L} \text { [26], [29] } \\
\text { - Sodium below } 125 \mathrm{mmol} / \mathrm{L} \text { [26] } \\
\text { - } \text { eGFR less than } 60 \text { [26], } \\
\text { - } \text { creatinine clearance[30] [31] } \\
\text { - } \text { CRP greater than } 50 \mathrm{mg} / \mathrm{L} \text { [26] } \\
\text { - } \text { previous hypoglycaemic episodes[32] [27] } \\
\text { - type of admission (emergency)[28] }\end{array}$ \\
\hline
\end{tabular}


Table 3. Unknown variables that may influence incidence of hypoglycaemia

\begin{tabular}{|l|l|l|l|}
\hline & Food intake & Exercise & Unknown errors \\
\hline $\begin{array}{l}\text { Reasons of data } \\
\text { unavailability }\end{array}$ & $\begin{array}{l}\text { Not recorded (or } \\
\text { only paper } \\
\text { records) during the } \\
\text { inpatient stay }\end{array}$ & $\begin{array}{l}\text { Not recorded } \\
\text { during the } \\
\text { inpatient stay }\end{array}$ & $\begin{array}{l}\text { Unknown drug } \\
\text { administration } \\
\text { error, unknown } \\
\text { physician/nurse } \\
\text { computer entry } \\
\text { error }\end{array}$ \\
\hline Possible mitigation & $\begin{array}{l}\text { Establish } \\
\text { electronic food } \\
\text { recording regimen }\end{array}$ & $\begin{array}{l}\text { Let diabetes } \\
\text { inpatients wear } \\
\text { easy-to-use } \\
\text { activity monitoring } \\
\text { systems such as } \\
\text { exercise } \\
\text { wristbands }\end{array}$ & $\begin{array}{l}\text { Extensive training } \\
\text { to medical staff to } \\
\text { increase the } \\
\text { awareness of the } \\
\text { potential risk faced } \\
\text { by diabetes } \\
\text { inpatients }\end{array}$ \\
\hline
\end{tabular}

Table 4. Preventative measures of inpatient hypoglycaemia

\begin{tabular}{|c|c|c|c|}
\hline Category & Human approach & $\begin{array}{l}\text { Computerised } \\
\text { approach }\end{array}$ & $\begin{array}{l}\text { Medical device } \\
\text { approach }\end{array}$ \\
\hline $\begin{array}{l}\text { Detailed } \\
\text { approach }\end{array}$ & $\begin{array}{ll}\text { - } & \text { Nurse/physicians } \\
& \text { training } \\
\text { - } & \text { Patient-education } \\
\text { - } & \text { New treatment } \\
\text { protocol/diabetes } \\
\text { service } \\
\text { - 'Hypoglycaemia } \\
\text { Committee' }\end{array}$ & $\begin{array}{ll}\text { - } & \text { Predictive } \\
\text { informatics } \\
\text { hypoglycae } \\
\text { mia risk-alert } \\
\text { system } \\
\text { - } & \text { Mobile client } \\
\text {-server } \\
\text { system for } \\
\text { computerize } \\
\text { d decision }\end{array}$ & $\begin{array}{ll}\text { - } & \text { Continuous } \\
\text { glucose } \\
\text { monitoring } \\
\text { devices } \\
\text { - } & \text { Insulin pumps } \\
\text { - } & \text { Closed-loop } \\
& \text { insulin delivery } \\
\text { ('artificial } \\
\text { pancreas') }\end{array}$ \\
\hline
\end{tabular}


Figure 1: Inpatients having one or more hypoglycaemic episode ${ }^{1}$ in last 7 days, England and Wales, 2011-17 (adapted from National Diabetes Inpatient Audit England and Wales, 2017 [9])

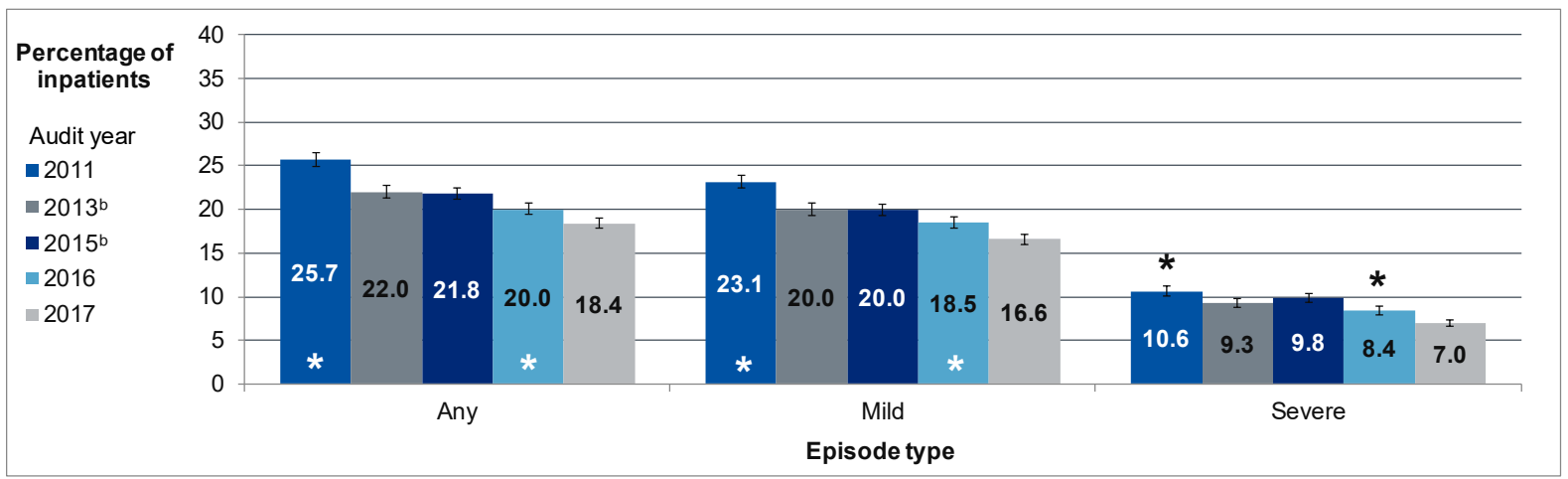

Notes:

$\mathbf{b}=$ break in time series.

* = statistically significant at the 0.05 level (vs. current audit year).

$\mathbf{n}=$ not statistically significant (vs. current audit year).

1. Any hypoglycaemic episode $=$ blood glucose measurement of $\leq 3.9 \mathrm{mmol} / \mathrm{L}$. Mild hypoglycaemic episode $=3.0-3.9 \mathrm{mmol} / \mathrm{L}$.

Severe hypoglycaemic episode $=<3.0 \mathrm{mmol} / \mathrm{L}$. 
Figure 2. Literature selection flow chart

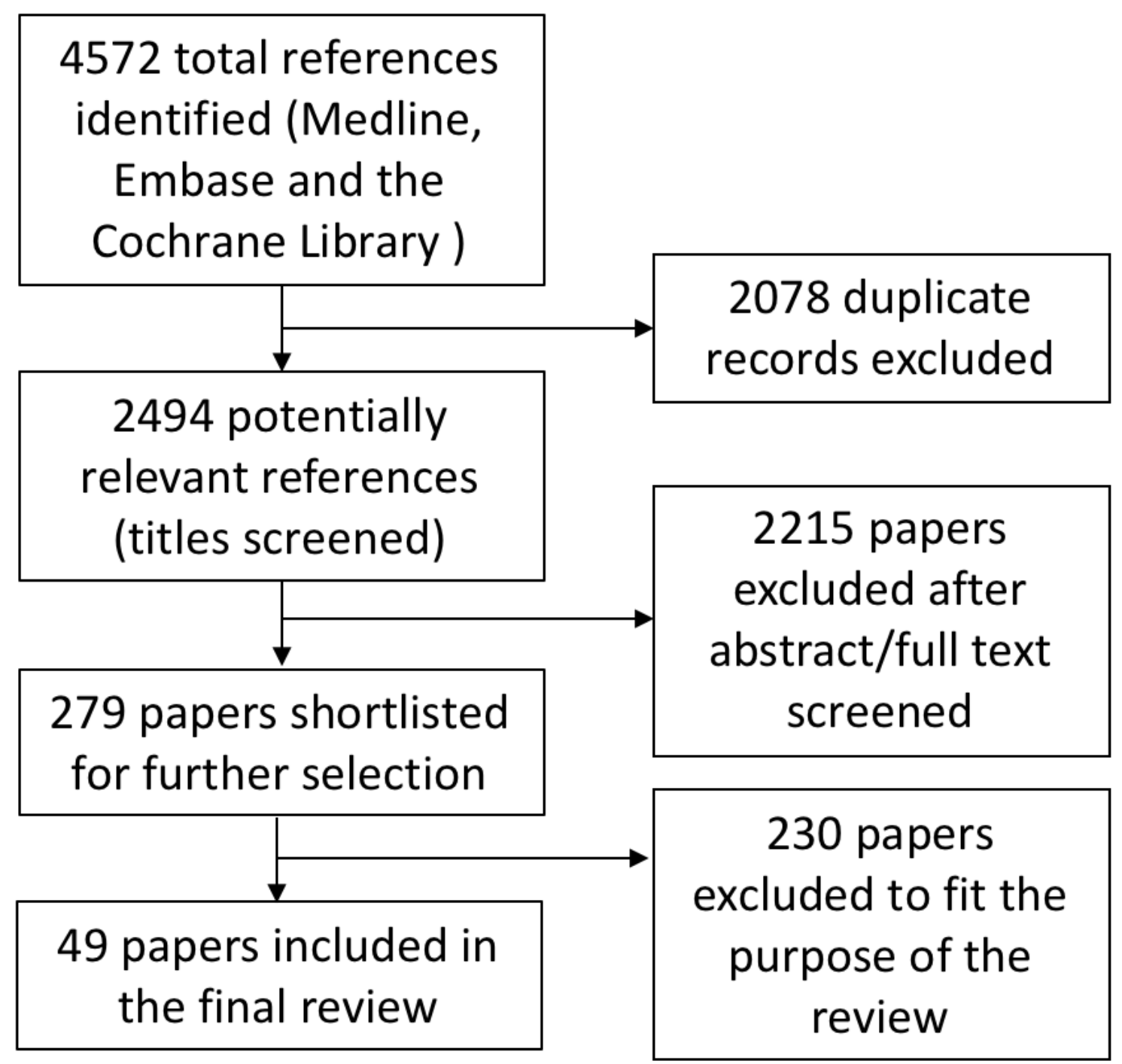




\section{References}

[1] G. E. Umpierrez, R. Hellman, M. T. Korytkowski, M. Kosiborod, G. A. Maynard, V. M. Montori, et al., "Management of hyperglycemia in hospitalized patients in noncritical care setting: an endocrine society clinical practice guideline.," J Clin Endocrinol Metab, vol. 97, pp. 16-38, Jan 2012.

[2] J. L. Schnipper, E. E. Barsky, S. Shaykevich, G. Fitzmaurice, and M. L. Pendergrass, "Inpatient management of diabetes and hyperglycemia among general medicine patients at a large teaching hospital.," J Hosp Med, vol. 1, pp. 145-50, May 2006.

[3] Anonymous, "Diabetes care in the hospital," Diabetes Care, vol. 40, pp. S120-S127, 01 Jan 2017.

[4] M. H. Murad, J. A. Coburn, F. Coto-Yglesias, S. Dzyubak, A. Hazem, M. A. Lane, et al., "Glycemic control in non-critically ill hospitalized patients: a systematic review and meta-analysis.," J Clin Endocrinol Metab, vol. 97, pp. 49-58, Jan 2012.

[5] R. M. Bailon, C. B. Cook, M. J. Hovan, B. P. Hull, K. M. Seifert, V. Miller-Cage, et al., "Temporal and geographic patterns of hypoglycemia among hospitalized patients with diabetes mellitus.," J Diabetes Sci Technol, vol. 3, pp. 261-8, Mar 2009.

[6] J. Giangola, K. Olohan, J. Longo, J. M. Goldstein, and P. A. Gross, "Barriers to hyperglycemia control in hospitalized patients: a descriptive epidemiologic study.," Endocr Pract, vol. 14, pp. 813-9, Oct 2008.

[7] T. Sein, T. T. N. Lau, H. C. Tan, and Y. K. D. Tay, "Hypoglycemia in non-critically ill patients with T2DM is independently associated with prolonged hospitalization," Diabetes, vol. 66, p. A585, June 2017.

[8] M. Evans, M. L. Wolden, B. L. Thorsted, P. C. McEwan, and J. L. Jacobsen, "Inpatient hypoglycaemia increases length of hospital stay and all-cause mortality risk," Diabetic Medicine, vol. 32, p. 23, March 2015.

[9] NHS. (2017). https://files.digital.nhs.uk/pdf/s/7/nadia-17-rep.pdf.

[10] B. J. Ulmer, A. Kara, and C. N. Mariash, "Temporal occurrences and recurrence patterns of hypoglycemia during hospitalization," Endocrine Practice, vol. 21, pp. 501-7, May 2015.

[11] A. Zapatero, R. Gomez-Huelgas, N. Gonzalez, J. Canora, A. Asenjo, J. Hinojosa, et al., "Frequency of hypoglycemia and its impact on length of stay, mortality, and shortterm readmission in patients with diabetes hospitalized in internal medicine wards," Endocrine Practice, vol. 20, pp. 870-5, Sep 2014.

[12] R. Gomez-Huelgas, R. Guijarro-Merino, A. Zapatero, R. Barba, A. Guijarro-Contreras, F. Tinahones, et al., "The frequency and impact of hypoglycemia among hospitalized patients with diabetes: A population-based study," Journal of Diabetes \& its Complications, vol. 29, pp. 1050-5, Nov-Dec 2015.

[13] E. Patel, A. Mallorie, R. Shamalathevy, D. Morgan, I. Akbarian, C. Psomadakis, et al., "Inpatient hypoglycaemia and national service framework for diabetes," Diabetic Medicine, vol. 33, p. 19, March 2016.

[14] V. Borzi and A. Fontanella, "The clinical impact of hypoglycemia in hospitalized patients," Italian Journal of Medicine, vol. 9, pp. 11-19, 2015.

[15] K. Nirantharakumar, T. Marshall, A. Kennedy, P. Narendran, K. Hemming, and J. J. Coleman, "Hypoglycaemia is associated with increased length of stay and mortality in people with diabetes who are hospitalized," Diabet Med, vol. 29, pp. e445-8, Dec 2012. 
[16] A. Turchin, M. E. Matheny, M. Shubina, S. V. Scanlon, B. Greenwood, and M. L. Pendergrass, "Hypoglycemia and clinical outcomes in patients with diabetes hospitalized in the general ward," Diabetes Care, vol. 32, pp. 1153-1157, July 2009.

[17] N. Yakubovich and H. C. Gerstein, "Serious cardiovascular outcomes in diabetes: the role of hypoglycemia," Circulation, vol. 123, pp. 342-8, Jan 252011.

[18] K. Malmberg, L. Ryden, H. Wedel, K. Birkeland, A. Bootsma, K. Dickstein, et al., "Intense metabolic control by means of insulin in patients with diabetes mellitus and acute myocardial infarction (DIGAMI 2): effects on mortality and morbidity," Eur Heart J, vol. 26, pp. 650-61, Apr 2005.

[19] A. Goyal, S. R. Mehta, R. Diaz, H. C. Gerstein, R. Afzal, D. Xavier, et al., "Differential clinical outcomes associated with hypoglycemia and hyperglycemia in acute myocardial infarction," Circulation, vol. 120, pp. 2429-37, Dec 152009.

[20] P. Akhavan, R. Aghili, M. Malek, A. Ebrahim Valojerdi, and M. E. Khamseh, "Hypoglycemia: Adverse Cardiovascular Outcomes in Non-Critically III People with Type 2 Diabetes," Arch Iran Med, vol. 19, pp. 82-6, Feb 2016.

[21] M. T. Korytkowski, "Hypoglycemia in patients with non-critical illness in the hospital setting," Diabetic Hypoglycemia, vol. 6, pp. 3-8, September 2013.

[22] M. Carey, L. Boucai, and J. Zonszein, "Impact of hypoglycemia in hospitalized patients," Current Diabetes Reports, vol. 13, pp. 107-13, Feb 2013.

[23] Y. Kim, K. B. Rajan, S. A. Sims, K. E. Wroblewski, and S. Reutrakul, "Hypoglycemia, not glycemic variability, predicts adverse hospital outcomes," Diabetes, vol. 62, p. A102, July 2013.

[24] Y. Kim, K. B. Rajan, S. A. Sims, K. E. Wroblewski, and S. Reutrakul, "Impact of glycemic variability and hypoglycemia on adverse hospital outcomes in non-critically ill patients," Diabetes Research \& Clinical Practice, vol. 103, pp. 437-43, Mar 2014.

[25] C. Fidler, T. Elmelund Christensen, and S. Gillard, "Hypoglycemia: an overview of fear of hypoglycemia, quality-of-life, and impact on costs," J Med Econ, vol. 14, pp. 64655, 2011.

[26] W. J. Katon, B. A. Young, J. Russo, E. H. Lin, P. Ciechanowski, E. J. Ludman, et al., "Association of depression with increased risk of severe hypoglycemic episodes in patients with diabetes," Annals of Family Medicine, vol. 11, pp. 245-50, May-Jun 2013.

[27] V. S. Eligar, P. Taylor, A. Tabasum, P. Nalla, A. Giannopoulou, S. Rice, et al., "Predicting inpatient hypoglycaemia risk with cardiff hypoglycaemia risk score (CHRIS)," Diabetes, vol. 66, pp. A102-A103, June 2017.

[28] K. Stuart, N. J. Adderley, T. Marshall, G. Rayman, A. Sitch, S. Manley, et al., "Predicting inpatient hypoglycaemia in hospitalized patients with diabetes: a retrospective analysis of 9584 admissions with diabetes," Diabetic Medicine, vol. 34, pp. 1385-1391, Oct 2017.

[29] I. Buziashvili, S. P. Klek, S. Sahansra, and L. E. Shapiro, "Hypoglycemic events in hospitalized patients with diabetes," Endocrine Reviews. Conference: 94th Annual Meeting and Expo of the Endocrine Society, ENDO, vol. 33, 2012.

[30] M. B. Elliott, S. J. Schafers, J. B. McGill, and G. S. Tobin, "Prediction and prevention of treatment-related inpatient hypoglycemia," Journal of Diabetes Science \& Technology, vol. 6, pp. 302-9, Mar 012012. 
[31] S. Schafers, M. Elliott, R. Reichley, J. Silverstein, J. McGill, and G. S. Tobin, "Prediction and prevention of inpatient hypoglycemia," Diabetes, vol. 60, pp. A136-A137, July 2011.

[32] N. S. Chevalier, P. Bohme, A. S. Durand-Lugger, A. Bassand, J. Vouillarmet, N. Marchant, et al., "Incidence, predictive factors and direct costs of severe hypoglycaemia: Results from the prospective HYPO.15 study," Diabetologia, vol. 1), pp. S459-S460, September 2015.

[33] F. Pasquel, S. Adeel, F. Farrokhi, I. Anzola, L. Peng, D. Smiley, et al., "Value of admission hemoglobin $\mathrm{A} 1 \mathrm{c}(\mathrm{HbA} 1 \mathrm{c})$ in predicting inpatient glycemic control and clinical outcome in non-ICU patients with type 2 diabetes," Diabetes, vol. 62, p. A222, July 2013.

[34] E. V. Varlamov, M. E. Kulaga, A. Khosla, D. L. Prime, and N. J. Rennert, "Hypoglycemia in the hospital: systems-based approach to recognition, treatment, and prevention," Hospital practice (1995) Hospital practice, vol. 42, pp. 163-72, Oct 2014.

[35] M. Anthony, "Hypoglycemia in hospitalized adults," MEDSURG Nursing, vol. 17, pp. 31-4, 40, Feb 2008.

[36] G. Marelli, F. Avanzini, G. lacuitti, E. Planca, I. Frigerio, G. Busi, et al., "Effectiveness of a nurse-managed protocol to prevent hypoglycemia in hospitalized patients with diabetes," Journal of Diabetes Research, vol. 2015 (no pagination), 2015.

[37] S. Asnani, L. Shvets, and C. Kaunzinger, "Reducing inpatient hypoglycemia: Diabetesa team approach," Journal of General Internal Medicine, vol. 27, pp. S536-S537, July 2012.

[38] S. Pasala, J. A. Dendy, V. Chockalingam, and R. Y. Meadows, "An inpatient hypoglycemia committee: Development, successful implementation, and impact on patient safety," Ochsner Journal, vol. 13, pp. 407-412, 2013.

[39] Y. Barak-Corren, R. Ben-Mayor, A. Hatsek, M. Sherman, and I. Hochberg, "Computer model for predicting hypoglycemia risk in diabetes patients in medical wards," Diabetes, vol. 64, p. A109, June 2015.

[40] K. Nirantharakumar, Y. F. Chen, T. Marshall, J. Webber, and J. J. Coleman, "Clinical decision support systems in the care of inpatients with diabetes in non-critical care setting: systematic review," Diabet Med, vol. 29, pp. 698-708, Jun 2012.

[41] C. R. Kilpatrick, M. B. Elliott, E. Pratt, S. J. Schafers, M. C. Blackburn, K. Heard, et al., "Prevention of inpatient hypoglycemia with a real-time informatics alert," Journal of Hospital Medicine (Online), vol. 9, pp. 621-6, Oct 2014.

[42] S. Spat, K. Donsa, P. Beck, B. Holl, J. K. Mader, L. Schaupp, et al., "A Mobile Computerized Decision Support System to Prevent Hypoglycemia in Hospitalized Patients With Type 2 Diabetes Mellitus," Journal of Diabetes Science \& Technology, vol. 11, pp. 20-28, Jan 2017.

[43] B. Buckingham, E. Cobry, P. Clinton, V. Gage, K. Caswell, E. Kunselman, et al., "Preventing hypoglycemia using predictive alarm algorithms and insulin pump suspension," Diabetes Technology \& Therapeutics, vol. 11, pp. 93-7, Feb 2009.

[44] M. Eren-Oruklu, A. Cinar, L. Quinn, and D. Smith, "Estimation of future glucose concentrations with subject-specific recursive linear models," Diabetes Technology \& Therapeutics, vol. 11, pp. 243-53, Apr 2009.

[45] F. Cameron, G. Niemeyer, K. Gundy-Burlet, and B. Buckingham, "Statistical hypoglycemia prediction," Journal of Diabetes Science \& Technology, vol. 2, pp. 61221, Jul 2008. 
[46] E. K. Spanakis, D. L. Levitt, T. Siddiqui, L. G. Singh, L. Pinault, J. Sorkin, et al., "The Effect of Continuous Glucose Monitoring in Preventing Inpatient Hypoglycemia in General Wards: The Glucose Telemetry System," Journal of Diabetes Science \& Technology, p. 1932296817748964, Dec 012017.

[47] F. Cameron, G. Niemeyer, D. M. Wilson, B. W. Bequette, K. S. Benassi, P. Clinton, et al., "Inpatient trial of an artificial pancreas based on multiple model probabilistic predictive control with repeated large unannounced meals," Diabetes Technology \& Therapeutics, vol. 16, pp. 728-34, Nov 2014.

[48] H. Thabit, S. Hartnell, J. M. Allen, A. Lake, M. E. Wilinska, Y. Ruan, et al., "Closed-loop insulin delivery in inpatients with type 2 diabetes: a randomised, parallel-group trial," The Lancet Diabetes \& Endocrinology, vol. 5, pp. 117-124, Feb 2017.

[49] H. Thabit and R. Hovorka, "Glucose control in non-critically ill inpatients with diabetes: towards closed-loop," Diabetes, Obesity \& Metabolism, vol. 16, pp. 500-9, Jun 2014.

[50] L. Bally, H. Thabit, S. Hartnell, E. Andereggen, Y. Ruan, M. E. Wilinska, et al., "ClosedLoop Insulin Delivery for Glycemic Control in Noncritical Care," N Engl J Med, Jun 25 2018.

[51] P. Akhavan, M. Malek, R. Aghili, A. Ebrahim Valojerdi, and M. E. Khamseh, "Hypoglycemia adversely affects short-term cardiovascular outcomes in noncritically ill people with type 2 diabetes initiating insulin therapy," Diabetes Technology and Therapeutics, vol. 17, p. A146, February 2015. 


\section{Conflict of interest}

YR, GDT, AL and RR declare no competing financial interests exist.

\section{Author contributions}

YR and RR wrote the manuscript. All authors critically reviewed the report.

\section{Acknowledgement}

YR is supported by a Novo Nordisk Postdoctoral Fellowship run in partnership with the University of Oxford.

The research was supported by the National Institute for Health Research (NIHR) Oxford Biomedical Research Centre (BRC). The views expressed are those of the authors and not necessarily those of the NHS, the NIHR or the Department of Health. 